\title{
MicroRNAs in cardiovascular disease: from pathogenesis to prevention and treatment
}

\author{
Daniel Quiat and Eric N. Olson
}

Department of Molecular Biology, University of Texas Southwestern Medical Center, Dallas, Texas, USA.

\begin{abstract}
The management of cardiovascular risk through lifestyle modification and pharmacotherapy is paramount to the prevention of cardiovascular disease. Epidemiological studies have identified obesity, dyslipidemia, diabetes, and hypertension as interrelated factors that negatively affect cardiovascular health. Recently, genetic and pharmacological evidence in model systems has implicated microRNAs as dynamic modifiers of disease pathogenesis. An expanded understanding of the function of microRNAs in gene regulatory networks associated with cardiovascular risk will enable identification of novel genetic mechanisms of disease and inform the development of innovative therapeutic strategies.
\end{abstract}

\section{Introduction}

In the early 20th century, cardiovascular disease surpassed infectious agents as the leading cause of mortality in the developed world (1). Ensuing epidemiological studies to uncover the etiology of heart disease significantly reduced patient mortality rates through the identification of diabetes, obesity, hypertension, and dyslipidemia as major, modifiable cardiovascular risk factors (2). In spite of these successes, cardiovascular diseases remain the predominant cause of morbidity and mortality worldwide (3). Today, knowledge of cardiovascular risk factors provides the means to proactively reduce patient death, but unfortunately also predicts an expanding pandemic of heart disease. This current and impending burden of disease underscores the need for further insight into the molecular mechanisms that contribute to the pathogenesis of cardiovascular disease and an expanded arsenal of therapeutic agents for prevention and treatment.

Disease phenotypes frequently involve the modulation of gene expression through non-coding RNAs (4). MicroRNAs (miRNAs) are short, non-coding nucleic acid regulators of mRNA stability and translation that play diverse roles in development and disease (5-7), and can be antagonized pharmacologically (8-10). In this Review, we focus on the in vivo role of miRNAs in the pathogenesis of several major risk factors for the development of cardiovascular disease, and their sequelae. We also highlight the potential for targeting miRNAs as preventative and reparative therapeutics for the treatment of cardiac disease.

\section{The biology of miRNAs}

miRNAs are highly conserved RNAs, 18-25 nucleotides in length, that regulate gene expression. miRNAs are encoded within the genome as intronic miRNAs, which are located in and processed from introns of protein-coding gene transcripts, or as intergenic miRNAs, which are transcribed under the control of their own promoters. Processing of the primary miRNA-encoding transcript in the nucleus by the RNase Drosha produces a pre-miRNA stem loop 80-110 nucleotides in length. Exportin 5 facilitates nuclear export of the pre-miRNA to the cytoplasm, where it is then processed into a mature miRNA/miRNA* duplex by Dicer (11). The

Conflict of interest: Eric N. Olson is a cofounder of miRagen Therapeutics, a biotechnology company developing microRNA-based therapeutics.

Citation for this article: J Clin Invest. 2013;123(1):11-18. doi:10.1172/JCI62876. mature miRNA is then loaded into the RNA-induced silencing complex (RISC) and dictates targeting of RISC to the 3 '-untranslated region (UTR) of mRNA transcripts. Recent data suggest that miRNAs primarily affect gene expression via subtle changes in mRNA transcript stability (12), thus resulting in small changes in protein levels (13). While the effects of any individual miRNA on a single target might be subtle, the combinatorial effects of a miRNA on multiple mRNA targets within a regulatory network can profoundly change the output of a pathway.

\section{Risk factors}

Hypertension. Arterial hypertension is defined by a consistent measurement of systolic blood pressure exceeding $140 \mathrm{mmHg}$ or diastolic blood pressure greater than $90 \mathrm{mmHg}$, with approximately $95 \%$ of clinical cases stemming from essential (primary) hypertension (14). Patients with hypertension have an increased lifetime risk of cardiovascular disease and heart failure $(15,16)$, and those with high-normal blood pressure similarly demonstrate a heightened risk (17). Though essential hypertension is idiopathic in nature, two hallmarks of the pathogenesis of this condition, and foci of current therapeutic strategies, are an increase in vascular tone and hyperactivation of the renin-angiotensin-aldosterone system (RAAS).

Vascular smooth muscle constitutes the medial layer of arteries and veins. The phenotype of a differentiated VSMC is plastic and can exhibit either proliferative or contractile properties that affect vascular function in normal and pathological settings (Figure 1 and ref. 18). miRNAs have been demonstrated to play an integral role in the transcriptional regulation of VSMC development, phenotype, and function during vascular pathology. Loss of miRNA processing via conditional deletion of Dicer specifically in embryonic or adult VSMCs results in embryonic lethality or loss of VSMC function, respectively, suggesting that miRNA expression is necessary for the development and maintenance of VSMCs $(19,20)$.

The smooth muscle-enriched miRNAs miR-143 and miR-145 are co-expressed, resulting from the transcriptional regulation of their bicistronic mRNA transcript by serum response factor and myocardin $(21,22)$. Genetic deletion of the miR-143/145 cluster in vivo demonstrates that these miRNAs are dispensable for smooth muscle specification; however, they are required for the transition between proliferative and contractile VSMC phenotypes. Consequently, mice lacking miR-143/145 display reduced arterial medial 


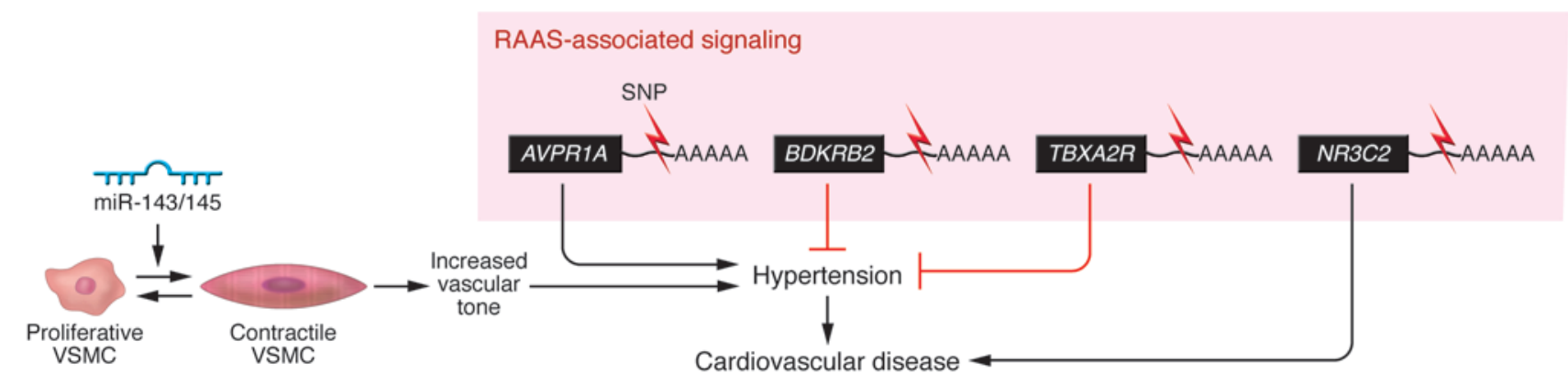

\section{Figure 1}

miRNA regulation of blood pressure. Pathological changes in vascular tone and RAAS signaling underlie primary arterial hypertension. miR-143 and miR-145 promote a contractile VSMC phenotype and are required for maintenance of normal vascular tone and RAAS-induced vasoconstriction. Human genome-wide association studies have identified SNPs in the miRNA binding sites of several RAAS-associated genes that correlate with a dysregulation of blood pressure.

thickness, decreased vascular tone, and reduced systemic blood pressure during homeostasis and following hypertensive challenge $(21,23)$. These in vivo effects of miR-143/145 are mediated by their destabilizing effect on transcripts encoding known repressors of the contractile VSMC phenotype, including Klf4, Klf5, and Ace.

Under normal physiological conditions, the RAAS hormonal axis functions to preserve fluid volume and arterial pressure through antinatriuretic and vasopressive effects. Pathological activation of RAAS is believed to be an underlying cause of essential hypertension. A recent linkage study demonstrated that SNPs in the miRNA binding sites of several RAAS-related genes are associated with alterations in blood pressure in humans (24). Patients with SNPs in the $3^{\prime}$ UTR of the vasopressin receptor $(A V P R 1 A)$ that lead to a loss of miR-526b and miR-578 binding exhibit increased blood pressure. In addition, several miRNA loss-of-function SNPs in potential miRNA binding sites of transcripts encoding the bradykinin 2 (BDKRB2) and thromboxane A2 $(T B X A 2 R)$ receptors are associated with reduced blood pressure. Intriguingly, an additional SNP in the $3^{\prime} \mathrm{UTR}$ of the aldosterone receptor (NR3C2) is not linked with alterations in blood pressure but is associated with an increased risk of myocardial infarction in young males (24). While the effect of these SNPs on the expression of their associated genes remains to be elucidated in vivo, their linkage to blood pressure phenotypes implicates miRNAs as possible modulators of RAAS signaling.

Hyperlipidemia. Elevated levels of serum cholesterol and other lipid molecules are broadly defined as hyperlipidemia. Clinically, total cholesterol measurements greater than $240 \mathrm{mg} / \mathrm{dl}$, with LDL fraction over $160 \mathrm{mg} / \mathrm{dl}$ and HDL under $40 \mathrm{mg} / \mathrm{dl}$, designate a state of dyslipidemia (25). The LDL/HDL ratio is a powerful indicator of cardiovascular risk $(26,27)$. Patients with elevated total or LDL cholesterol are at greater risk for coronary heart disease (28), while those patients with serum HDL levels in the 80th percentile have a significantly reduced risk of cardiovascular disease (29).

Regulation of serum lipid levels by the liver is influenced by hepatic miRNA content (Figure 2). Liver-specific genetic ablation of Dicer-mediated miRNA processing results in aberrant gene expression and metabolism (30). These changes are associated with increased levels of free serum cholesterol and fatty acids, as well as an increase in the concentration of cholesterol, fatty acids, and triglycerides in the liver. Together, these data suggest that hepatic miRNA expression is required for normal lipid homeostasis.
The transcriptional activator SREBP is a master regulator of endogenous sterol biosynthesis (31). In humans, the primary mRNA transcripts encoding SREBP1 and SREBP2 proteins also encode miR-33b and miR-33a, respectively. miR-33a/b negatively regulate the membrane transporter $A B C A 1$ to reduce cholesterol efflux $(32,33)$ and target the transcripts CROT, CPT1a, HADHB, and $A M P K a$ to dampen the $\beta$-oxidation of fatty acids (34). Thus, intronically encoded miR-33a/b collaborate with their protein coding host transcript to promote the cholesterologenic state (Figure 2). Antisense inhibition of the singular miR-33 species in mice increases total and HDL serum cholesterol concentrations, consistent with increased cellular cholesterol export via the upregulation of ABCA1 in macrophages and hepatocytes $(32,33)$. Subsequent investigation of anti-miR-33a/b therapy in primates demonstrated a lowering of serum VLDL triglyceride levels and an increase in HDL cholesterol, suggesting that antagonism of miR-33 might beneficially alter a patient's serum lipid profile (35).

miR-122 is one of the most abundantly expressed miRNAs in the liver $(36,37)$ and is specifically enriched in hepatocytes (38). This hepatic enrichment makes miR-122 highly amenable to pharmacological inhibition by various anti-miR chemistries that are efficiently distributed to the liver. Two studies have demonstrated that antagonism of miR-122 in mice, with various antimiR chemistries, reduces total serum cholesterol levels $(8,39)$. These studies reveal that inhibition of miR-122 leads to the de-repression of a multitude of transcripts with miR-122 seed matches, resulting in increased fatty acid oxidation and reduced lipid synthesis in the liver. Further development of locked nucleic acid-modified (LNA-modified) anti-miR-122 therapies, primarily as antiviral therapy against hepatitis $C$ virus infection, have similarly revealed the beneficial effects of anti-miR-122 treatment on lipid profiles in primates and established proof of efficacy and minimal toxicity of LNA-modified anti-miR therapeutics in a preclinical model $(40,41)$.

Obesity and diabetes. Type II diabetes is characterized by a dysregulation of blood glucose levels and results from progressive peripheral insulin resistance in conjunction with pathologically altered pancreatic insulin secretion. Diabetes is diagnosed by repeated measurement of fasting glucose levels over $126 \mathrm{mg} / \mathrm{dl}$, or over $200 \mathrm{mg} / \mathrm{dl}$ following oral glucose challenge, and type II diabetes often presents in obese, inactive individuals. Men and women with diabetes have an increased risk for cardiovascular 


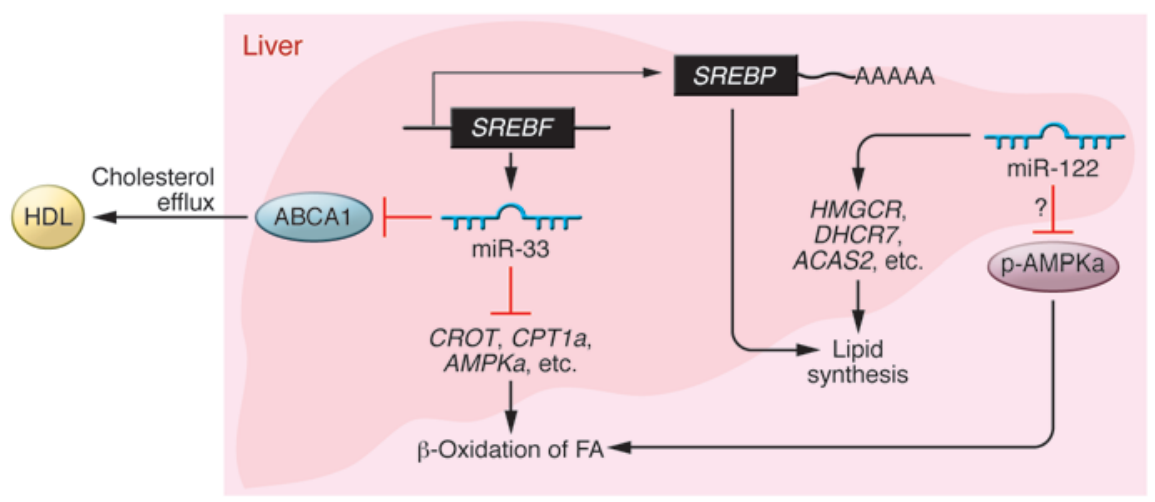

\section{Figure 2}

Role of miR-33 and miR-122 in hepatic lipid metabolism. The SREBF locus encodes the lipogenic transcription factor SREBP and miR-33, which represses mRNA transcripts of genes in the fatty acid (FA) $\beta$-oxidation and cholesterol efflux pathways. miR-122 promotes lipid synthesis by supporting the expression of lipid biosynthetic genes, and dampens fatty acid oxidation by inhibiting phosphorylation of AMPKa by an unknown mechanism. disease (42), and the frequent comorbidity of type II diabetes with other cardiovascular risk factors, such as hypertension, dyslipidemia, and obesity, significantly increases the risk of heart failure in these patients (16).

miRNA processing is required for proper pancreatic endocrine function. Deletion of Dicer in the developing pancreas inhibits pancreatic islet formation and insulin-secreting $\beta$ cell differentiation, indicating the importance of miRNAs in lineage specification of the endocrine pancreas (43). Removal of Dicer in mature $\beta$ cells using an insulin promoter-driven Cre-recombinase does not affect $\beta$ cell number, suggesting that miRNAs are not required for maintenance of differentiated $\beta$ cells. However, this study did not interrogate the function of adult $\beta$ cells lacking mature miRNAs. Additionally, miRNAs are integral to the function of fat-storing adipocytes, which are central to the pathology of obesity and type II diabetes. Conditional ablation of Dicer in the adipocyte lineage results in decreased fat mass and lipogenesis in white but not brown fat depots (44).

miR-375 is a $\beta$ cell-enriched miRNA whose expression is elevated in the pancreatic islets of $o b / o b$ diabetic mice and patients with type II diabetes (45-47). Pharmacological studies have revealed that miR-375 negatively regulates the exocytosis of insulin by targeting myotrophin, as treatment with anti-miR-375 antagomir in mice improves insulin secretion (45). Genetic interrogation of miR-375 function revealed that mice lacking miR-375 are viable, exhibit reduced $\beta$ cell mass, develop hyperglycemia under normal conditions, and demonstrate exacerbated hyperglycemia in the context of a genetic $(o b / o b)$ model of diabetes (47). This phenotype can be attributed to the de-repression of a constellation of growth inhibitory genes in the absence of miR-375. Together, these data suggest that dysregulation of miR-375 in the setting of diabetes is a compensatory mechanism to increase $\beta$ cell mass in order to counteract increased peripheral insulin resistance.

The expression of miR-103 and miR-107 is elevated in the liver of rodent models of obesity and type II diabetes $(48,49)$. The miRNAs miR-103 and miR-107 differ by only 1 nucleotide $3^{\prime}$ of their seed region and constitute a miRNA family. Adenoviral-mediated overexpression of miR-107 in the liver leads to hyperglycemia, hyperinsulinemia, and an increase in hepatic gluconeogenesis. In contrast, pharmacological inhibition of miR-103/107 with antimiR-103 antagomir improves glucose homeostasis in obese mice, reduces adipocyte size, and decreases overall fat mass (49). Pharmacological inhibition of miR-103 potentiates insulin signaling via the upregulation of the target gene caveolin-1 and its subsequent stabilization of the insulin receptor (49). This modulation of upstream insulin signaling components suggests that antimiR-103 could be used to therapeutically sensitize peripheral tissue to insulin signaling in the diabetic state.

The miR-143/145 cluster is similarly upregulated in the liver of diabetic rodents (50). Overexpression of miR-143 in the liver is sufficient to drive glucose intolerance and insulin resistance, while knockout mice lacking miR-143/145 display reduced obesity-induced insulin resistance. These phenotypic effects are associated with dampened or heightened activation of the AKT pathway, respectively. The effect of miR-143 in glucose homeostasis is mediated by the target oxysterol binding protein-like 8 (ORP8), which has been shown to have a novel role in promoting AKT phosphorylation (50).

The let-7 family of miRNAs was first identified through forward genetics as a regulator of developmental timing in C. elegans (51), but has been shown to exert a powerful effect on growth and metabolism in mammals (Figure 3A). Global transgenic overexpression of Lin28a, a negative regulator of let-7 biogenesis, significantly increases the growth and organ size of mice and markedly reduces age-associated and high-fat diet-induced obesity (52). Conversely, ubiquitous overexpression of let-7 at modest levels results in reduced body size and aberrant glucose homeostasis, leading to hyperglycemia $(53,54)$. Conditional overexpression of let- 7 in the pancreas is sufficient to reduce insulin secretion (54). The effects of let-7 on peripheral insulin sensitivity were identified by deletion of Lin28a and the concomitant elevation of let-7 specifically in skeletal muscle, which was sufficient to induce a hyperglycemic phenotype in mice (53). Additionally, knockdown of the let-7 family with a LNA-modified anti-miR reduces peripheral insulin resistance induced by high-fat diet (54). In these studies, the metabolic effects of the Lin28/let-7 regulatory cascade were attributed to let-7 targeting of the insulin receptor (Insr) and its downstream mediators, such as Irs 2 and Igf1r, in skeletal muscle and liver. Finally, Zhu et al. showed in large-scale genome wide association studies that multiple let-7 targets are associated with type II diabetes (53), suggesting a potential role for aberrant regulation of let- 7 target genes in the diabetic patient. Together, these studies indicate that let- 7 acts to decrease insulin secretion centrally and induce insulin resistance in peripheral metabolic tissues such as skeletal muscle. Of particular interest is the ability of short-term anti-let-7 administration to reduce blood glucose levels by decreasing peripheral insulin resistance, suggesting anti-let-7 therapy as a potential insulin-sensitizing agent (54).

The continuous contractile mechanics of the heart demand a high amount of substrate for energy production and are sensitive to metabolic derangements (55). mRNA transcripts coding for 
A

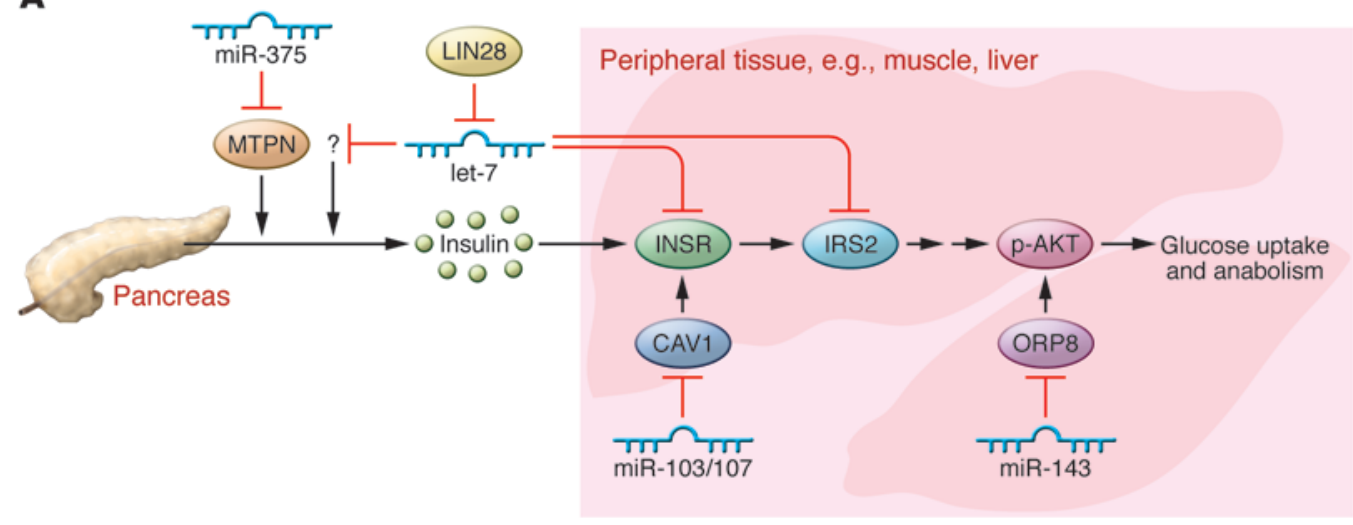

B

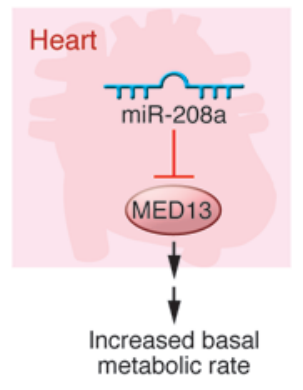

Figure 3

miRNA modulation of insulin signaling and metabolic homeostasis. (A) miRNAs regulate pancreatic insulin secretion and the insulin signaling cascade in peripheral tissues. Pathological changes in the expression of these miRNAs contribute to the type II diabetic phenotype. (B) Modulation of Med13 expression in the heart by the cardiac-specific miRNA, miR-208a, regulates whole body metabolism and adiposity.

three cardiac myosin heavy chain contractile proteins, $M y b 6, M y b 7$, and Myb7b, encode miRNAs miR-208a, miR-208b, and miR-499, respectively, within their introns (56). These miRNAs function redundantly to control cardiac contractility in response to pathological hypertrophic remodeling and thyroid hormone signaling $(57,58)$. This action is mediated in part by MED13, a component of the mediator transcriptional complex (58). Acute inhibition of miR-208a with LNA-modified anti-miR leads to the upregulation of Med13 transcript in the heart and significantly reduces weight gain in mice that are fed a high-fat diet (Figure 3B and ref. 59). Transgenic overexpression of Med13 specifically in the murine heart increases the basal metabolic rate, as measured by $\mathrm{O}_{2}$ consumption and $\mathrm{CO}_{2}$ production, and reduces overall fat mass on normal- and high-fat diets. In contrast, conditional deletion of Med13 in the heart exacerbates weight gain in mice fed a high-fat diet. This study demonstrates the influence of a cardiac miRNA family on whole-body metabolism via the regulation of Med13 and suggests a novel mechanism by which cardiac contractility and energy demand interface with global energy homeostasis. Given the multiplicity of miR-208 target transcripts, additional target genes likely contribute to this regulatory pathway.

\section{Sequelae}

Atherosclerosis. The prolonged pathogenesis of atherosclerosis is marked by endothelial cell dysfunction, followed by deposition of lipid-laden macrophages and VSMC proliferation, leading to occlusion of the arterial lumen. Hypertension and hyperlipidemia significantly contribute to the process of atherosclerotic plaque formation (60), as do the miRNAs that regulate these processes. The positive regulation of cholesterol efflux by the miR-33 family promotes atheroma formation. Treatment of hypercholesterolemic Ldlr-null mice with an anti-miR-33 compound causes a regression of atherosclerotic lesions, likely due to the regulation of ABCA1 in resident plaque macrophages (61).

Gain and loss of function of smooth muscle-enriched miR-143/145 in vivo results in reduced smooth muscle cell proliferation and consequently prevents neointima formation in surgical models of vascular injury, suggesting that closely titrated levels of miR-143/145 are required for the pathological proliferative response to injury
$(21,62)$. Similarly, antisense oligonucleotide inhibition of the broadly expressed miR-21 reduces neointima formation following vascular balloon injury via increased levels of the pro-apoptotic proteins BCL2 and PTEN (63).

miRNAs also appear to mediate direct anti-atherogenic signals between healthy endothelial cells and vascular smooth muscle (64). Normal laminar blood flow induces transcriptional activation of miR-143/145 by KLF2 in endothelial cells, where these miRNAs are packaged into vesicles and secreted to neighboring vascular smooth muscle to support contractile gene expression (Figure 4A). miR-143/145-enriched vesicles from endothelial cells reduce atherosclerotic plaque size in $\mathrm{Apoe}^{-/-}$mice. In the setting of endothelial cell injury, this miRNA-mediated communication is disrupted, leading to a proliferative VSMC phenotype in the absence of endothelial-derived miR-143/145.

miR-126 is encoded within an intron of Egfl7 and is enriched in endothelial cells $(65,66)$. Genetic deletion of miR-126 disrupts vascular integrity and results in partial embryonic lethality. miR-126 knockout mice that survive to adulthood demonstrate defective neo-angiogenesis following a surgical model of myocardial infarction. miR-126 is enriched in the apoptotic bodies of dying endothelial cells and signals in a paracrine manner to induce the anti-apoptotic, pro-repair, CXCL12-CXCR4 signaling cascade in neighboring vascular cells (67). Direct administration of premiR-126, or infusion of miR-126-containing apoptotic bodies, respectively decreases the size or promotes the stabilization of atherosclerotic lesions in Apoe $\mathrm{A}^{-/-}$mice.

Cardiac ischemia. Atherosclerotic plaque formation in the coronary vasculature impedes blood flow, resulting in transient bouts of cardiomyocyte ischemia, and often presents clinically as angina. Cardiomyocytes possess inducible protective mechanisms to dampen injury associated with ischemia and infarction (68), and a thorough understanding of miRNA involvement in these intrinsic defense mechanisms may provide new therapeutic targets to reduce injury following myocardial infarction.

miRNAs are dynamically regulated in rodent models of ischemia/reperfusion injury and infarction, as well as in cardiac biopsies from human subjects following myocardial infarction $(69,70)$. miR-214 is significantly upregulated in the heart following ischemia 
A

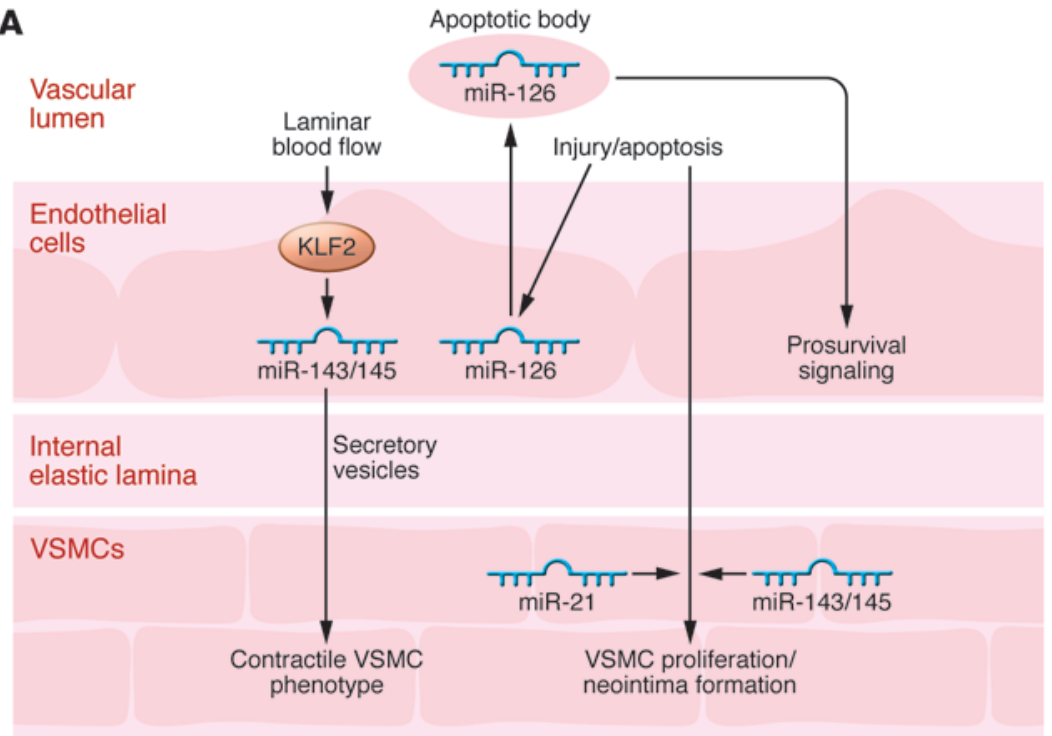

B

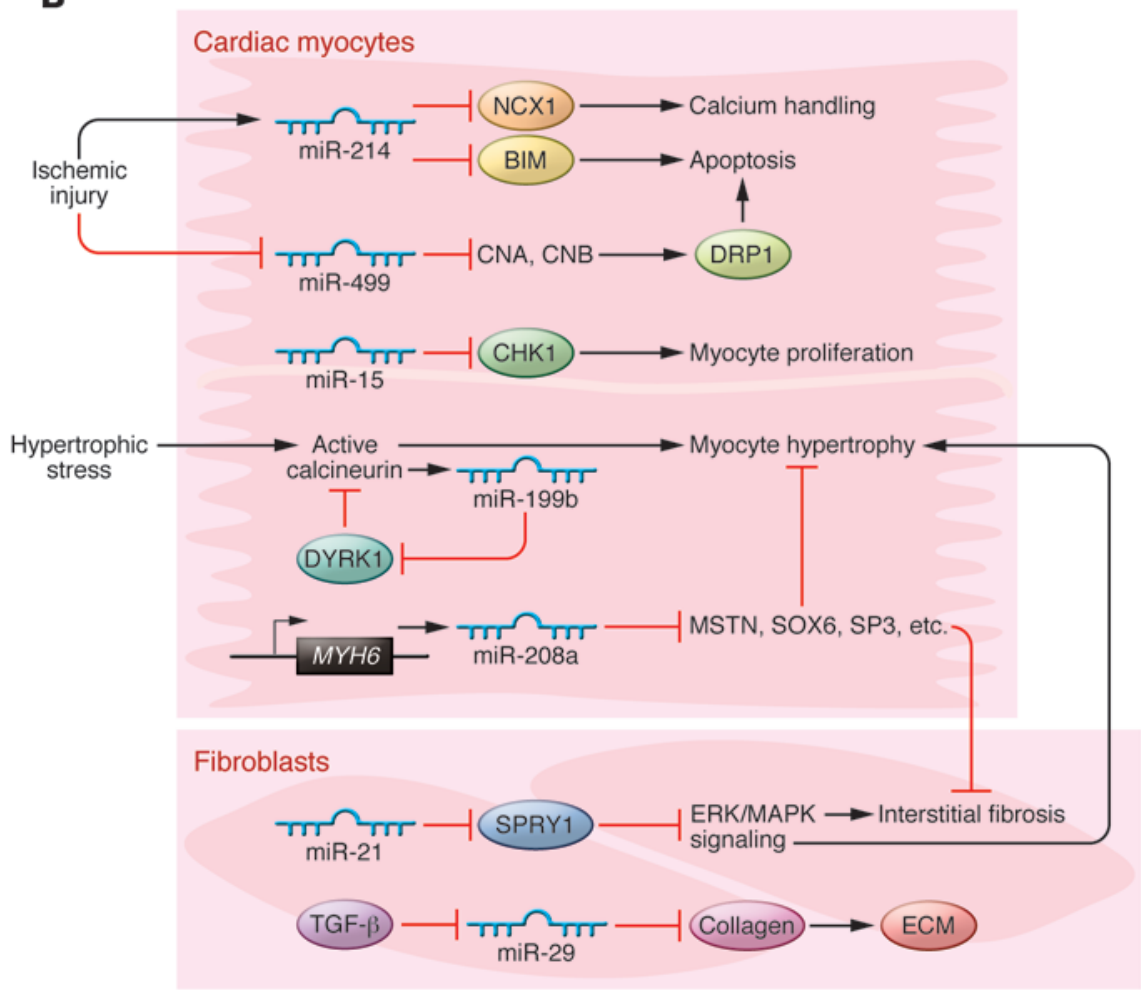

Figure 4

miRNAs in cardiovascular pathology. (A) miRNAs in endothelial cells act as paracrine signals to preserve vascular structure under normal conditions and to promote cell survival following injury. VSMC miRNAs modulate the smooth muscle response to injury. These mechanisms are dysregulated during atherosclerosis. (B) Cardiomyocyte and fibroblast miRNAs regulate cellular survival and pathological responses to multiple cardiac stressors.

(Figure 4B and refs. 69, 71). In mice, deletion of miR-214 exacerbates injury following myocardial ischemia/reperfusion and increases mortality following myocardial infarction (71). In the pathology of ischemia/reperfusion injury, miR-214 attenuates calcium overload by titrating the expression of the sodium/calcium exchanger NCX1 in cardiomyocytes and promotes cell survival by inhibiting expression of the pro-apoptotic protein BIM.

The expression of myosin heavy chain-encoded miR-499 is downregulated in response to hypoxic or ischemic stress in cardiomyocytes (72). Transgenic overexpression of miR-499 blunts cardiomyocyte apoptosis and infarct size following ischemia/reperfusion injury, while knockdown of miR-499 with a cholesterol-modified antagomir promotes myocardial apoptosis and broadens the infarct zone (72). This cardioprotective effect of miR-499 results, at least in part, from its targeting of the $\alpha$ - and $\beta$-isoforms of calcineurin, a central regulator of pathological cardiac remodeling (73), and their downstream effects on dynamin-related protein 1 induction of mitochondrial fission and apoptosis. Of note, elevated miR-499 levels prime the heart for dysfunction in response to hypertrophic stress signals (74, $75)$, suggesting that acute but not chronic elevation of miR-499 might be of therapeutic interest for cardioprotection from ischemia and infarction.

Cardiac remodeling. Cardiac diseases of various origin are linked by their common histological features of myocyte death, associated with compensatory pathological remodeling and minimal functional repair. miRNAs are intimately involved in cardiac repair and remodeling responses to injury, and their ablation in the adult heart, either by conditional or temporal deletion of Dicer or Dgcr8, is sufficient to drive heart failure and death $(76,77)$.

Increased contractile demand in the setting of systemic hypertension, or in response to loss of neighboring viable myocytes drives pathological cardiomyocyte hypertrophy and interstitial fibrosis. This compensatory hypertrophic response preserves cardiac output in the pathological setting and involves a signature pattern of miRNA dysregulation $(78,79)$.

miR-21 is widely expressed and is one of the most significantly upregulated miRNAs in rodent models of cardiac hypertrophy (78). Pharmacological antagonism of miR-21 with antagomir exhibits antihypertrophic and antifibrotic effects, resulting in functional improvement following hypertrophic stress (80). This therapeutic effect has been attributed to reduced activation of the ERK/ MAPK signaling cascade in cardiac fibroblasts, resulting from the upregulation of the miR-21 target Sprouty1. Intriguingly, genetic ablation of miR-21 or inhibition with 8-mer LNAs are not sufficient to blunt the hypertrophic and fibrotic responses to a 
variety of cardiac stress stimuli (81). These fundamental differences between the pathological response following genetic loss or pharmacological inhibition of miR-21 might reflect unique dosage effects of miR-21, differences between constitutive and temporal loss of miR-21, differential uptake of miR-21 inhibitors in cardiac fibroblasts versus cardiomyocytes, or off-target effects of antagomir-21. Additionally, alternate conclusions from these miR-21 loss-of-function studies might be explained in part by the use of different anti-miR chemistries that vary in nucleotide length and chemical composition.

The miR-199 family of miRNAs is similarly upregulated in genetic and surgical rodent models of cardiac hypertrophy $(78,79)$. miR-199b is induced by, and positively regulates, the pro-hypertrophic calcineurin/NFAT signaling cascade by inhibiting expression of DYRK1 kinase, thus forming a feed-forward mechanism that potentiates pathological growth (82). Inhibition of miR-199b with antagomir increases the expression of DYRK1 and blunts or reverses pathological remodeling.

miR-208a is encoded by the $\alpha$-myosin heavy chain gene (Myb6) transcript and dominantly regulates the expression of the related miRNAs miR-208b and miR-499. Loss of miR-208a results in loss of miR-208b/499 expression in the heart, and prevents pathological hypertrophic remodeling and fibrosis (56-58). miR-208a regulation of myocyte hypertrophy and contractile protein expression occurs through the target genes myostatin, Sox6, Purb, Sp3, and $H p 1 b$. Inhibition of miR-208a by injection of LNA-anti-miR prevents cardiac remodeling and improves cardiac function and survival in a Dahl rat hypertensive model of cardiac hypertrophy (83).

The cardiac response to infarction injury is characterized by coagulative necrosis of myocytes, followed by extracellular matrix deposition and scar formation by cardiac fibroblasts. Myocardial infarction is associated with a decrease in levels of miR-29 family members, allowing for the expression and deposition of collagen components of the fibrotic scar (84). Conversely, heightened miR-29 expression is associated with reduced vascular stromal integrity, and anti-miR-29 therapy stabilizes extracellular matrix, preventing aortic dilation and aneurysm formation $(85,86)$. These in vivo functions for miR-29 highlight the fragile balance of beneficial and pathological fibrotic deposition that should be considered when developing miR-29 therapies.

Cardiomyocyte regeneration. Though the heart has limited regenerative capacity, recent evidence suggests that replacement of cardiomyocytes can occur through the proliferation and migration of existing cardiomyocytes (87-89). The miR-15 family of miRNAs, composed of miR-15a/b, miR-16-1/2, miR-195, and miR-497, contain a homologous sequence at their seed regions and exhibit marked upregulation coinciding with the last wave of cardiomyocyte proliferation in the postnatal heart (90). The miR-15 family promotes mitotic cell cycle exit by diminishing the expression of checkpoint kinase 1 and other promitotic genes. Antagonism of all miR-15 family members with a single inhibitory chemistry increases myocyte proliferation in hearts of neonatal mice, prevents ischemic damage in the adult mouse heart, and improves cardiac function following myocardial infarction $(90,91)$.

\section{Translating miRNA biology to therapeutics}

The preceding studies convincingly implicate miRNAs in the pathogenesis of multiple conditions that lead to the development of cardiovascular disease and the subsequent cardiac response to the diseased state. The robust functions of miRNAs in these pathways present unique challenges to incorporating pharmacological targeting of miRNAs into patient treatment. The following examples highlight these obstacles.

Preventative miRNA therapies. The role of miRNAs in the pathogenesis of the aforementioned cardiovascular risk factors suggests that they might be interesting candidate drug targets. The administration of miRNA-targeted therapies for long-term risk modification warrants the consideration of several potential adverse effects.

Many of the miRNAs involved in cardiovascular pathology have also been implicated as tumor suppressors in vivo (92). For example, the let-7 family of miRNAs, which can be antagonized to induce peripheral insulin sensitivity, functions to promote differentiation and suppress tumor initiation, and is downregulated in a variety of malignancies (93). Thus, the potential use of anti-let-7 therapy to counteract the diabetic state should take into consideration the long-term risk of developing cancer. Notably, tumorigenic effects of let-7 inhibition have not been reported, though in vivo evidence has demonstrated the antitumor affect of let-7 in gain-of-function experiments (94). These concerns might warrant development of targeted let-7 antagonism specifically in skeletal myofibers. Furthermore, let-7 inhibition in vivo promotes $\beta 2$-adrenergic receptor expression and may complicate the use of $\beta$-adrenergic antagonists in patients with cardiovascular disease (95).

Other undesirable side effects of chronically administered miRNA therapeutics could be mitigated by modulation of miRNA expression in a particular tissue of interest. This could be achieved by targeted delivery of miRNA therapeutics or specifically targeting miRNAs that display restricted expression in a tissue of interest and/or specific regulation of a biochemical process. Chronic inhibition of miR-122 and miR-33a/b, hepatic and cholesterol metabolism-specific miRNAs, respectively, might beneficially alter serum lipids and may be less likely to provoke undesirable side effects. Additionally, targeting of $\beta$ cell-enriched miRNAs to increase insulin secretion could aid in glycemic control in diabetic patients. Finally, chronic antagonism of smooth muscle-enriched miR-143/145 to treat essential hypertension and confer metabolic improvement in diabetic patients would ideally result in minimal on-target effects in other tissues. This therapeutic strategy may minimize undesirable off-tissue effects but does not address the potential adverse on-target effects of chronic miRNA antagonism in the cell type of interest.

Reparative miRNA therapies. The biologically diverse functions of miRNAs underscore the need for targeted delivery and desirable pharmacokinetic profiles of inhibitory and mimic miRNA chemistries for reparative therapeutics. Acute miR-15 antagonism might confer benefits to cardiac repair and function following myocardial infarction by inducing myocyte proliferation, while prolonged anti-miR-15 therapy could potentially induce global cellular proliferation and promote neoplasia. Targeted delivery of anti-miR-15 might circumvent these adverse systemic effects, as might rapidly metabolized chemistries that transiently inhibit miR-15 following myocardial infarction.

The therapeutic manipulation of the miR-29 family presents a similar requirement for targeted therapy. In the context of postmyocardial infarction remodeling, overexpression of miR-29 might beneficially reduce the extent of scar formation. However, such treatment might disrupt the vascular integrity of the aorta. Again, local delivery of molecules that mimic miR-29, possibly via drugeluting coronary stents, could prevent such adverse outcomes. 
The outlook for the clinical application of miRNA discoveries is promising. miRNA diagnostics and therapeutics, such as the use of cardiovascular miRNA biomarkers (96) and ongoing phase II clinical trails of miRNA inhibitory molecules (97), exemplify this potential. Additionally, a breadth of intriguing in vitro data implicate miRNAs in many more cardiovascular disease processes and pathways, but the role of miRNAs remains to be tested in vivo in disease models. In conclusion, the involvement of miRNAs in cardiovascular risk factor pathology and subsequent cardiovascular disease warrant further genetic and pharmacological elucidation of their function in vivo in order to support the development of miRNA and gene regulatory therapies for the prevention and treatment of cardiovascular disease.

\section{Acknowledgments}

We thank Ning Liu and Robert Frost for insightful comments and José Cabrera for help with graphics. E. Olson is supported by grants from the NIH, the Leducq Foundation, the Robert A. Welch Foundation, and the American Heart Association: Jon Holden DeHaan Foundation. D. Quiat is supported by a Kirschstein $\mathrm{MD} / \mathrm{PhD}$ fellowship from the National Heart, Lung, and Blood Institute (1F30HL103013).

Address correspondence to: Eric N. Olson, Department of Molecular Biology, University of Texas Southwestern Medical Center, 5323 Harry Hines Boulevard, Dallas, Texas 75390-9148, USA. Phone: 214.648.1187; Fax: 214.648.1196; E-mail: Eric.Olson@ utsouthwestern.edu.
1. Cohen ML. Changing patterns of infectious disease. Nature. 2000;406(6797):762-767.

2. National Heart, Lung, and Blood Institute. Chapter 4: Disease statistics. NHLBI Fact Book, Fiscal Year 2011. NIH Web site. http://www.nhlbi.nih.gov/about/ factbook/chapter4.htm. Accessed May 18, 2012.

3. WHO. Causes of Death 2008 Summary Tables. Geneva, Switzerland: World Health Organization; 2008.

4. Mercer TR, Dinger ME, Mattick JS. Long non-coding RNAs: insights into functions. Nat Rev Genet. 2009;10(3):155-159.

5. Small EM, Olson EN. Pervasive roles of microRNAs in cardiovascular biology. Nature. 2011; 469(7330):336-342.

6. Chang TC, Mendell JT. microRNAs in vertebrate physiology and human disease. Annu Rev Genomics Hum Genet. 2007;8:215-239.

7. Mendell JT, Olson EN. 2012. MicroRNAs in stress signaling and human disease. Cell. 2012; 148(6):1172-1187.

8. Krutzfeldt J, et al. Silencing of microRNAs in vivo with 'antagomirs'. Nature. 2005;438(7068):685-689.

9. Orom UA, Kauppinen S, Lund AH. LNA-modified oligonucleotides mediate specific inhibition of microRNA function. Gene. 2006;372:137-141.

10. Obad S, et al. Silencing of microRNA families by seed-targeting tiny LNAs. Nat Genet. 2011; 43(4):371-378.

11. Bartel DP. MicroRNAs: genomics, biogenesis, mechanism, and function. Cell. 2004;116(2):281-297.

12. Guo H, Ingolia NT, Weissman JS, Bartel DP. Mammalian microRNAs predominantly act to decrease target mRNA levels. Nature. 2010;466(7308):835-840.

13. Baek D, Villén J, Shin C, Camargo FD, Gygi SP, Bartel DP. The impact of microRNAs on protein output. Nature. 2008;455(7209):64-71.

14. Carretero OA, Oparil S. Essential hypertension. Part I: definition and etiology. Circulation. 2000; 101(3):329-335.

15. Kannel WB. Blood pressure as a cardiovascular risk factor: prevention and treatment. JAMA. 1996; 275(20):1571-1576.

16. He J, Ogden LG, Bazzano LA, Vupputuri S, Loria C, Whelton PK. Risk factors for congestive heart failure in US men and women: NHANES I epidemiologic follow-up study. Arch Intern Med. 2001; 161(7):996-1002.

17. Vasan RS, et al. Impact of high-normal blood pressure on the risk of cardiovascular disease. $N$ Engl J Med. 2001;345(18):1291-1297.

18. Owens GK. Regulation of differentiation of vascular smooth muscle cells. Physiol Rev. 1995; 75(3):487-517.

19. Albinsson S, Suarez Y, Skoura A, Offermanns S, Miano JM, Sessa WC. MicroRNAs are necessary for vascular smooth muscle growth, differentiation, and function. Arterioscler Thromb Vasc Biol. 2010; 30(6):1118-1126.

20. Albinsson S, et al. Smooth muscle miRNAs are critical for post-natal regulation of blood pressure and vascular function. PLoS One. 2011;6(4):e18869.

21. Xin M, et al. MicroRNAs miR-143 and miR-145 modulate cytoskeletal dynamics and responsiveness of smooth muscle cells to injury. Genes Dev. 2009; 23(18):2166-2178.

22. Cordes KR, et al. miR-145 and miR-143 regulate smooth muscle cell fate and plasticity. Nature. 2009; 460(7256):705-710

23. Boettger $\mathrm{T}$, et al. Acquisition of the contractile phenotype by murine arterial smooth muscle cells depends on the Mir143/145 gene cluster. J Clin Invest. 2009;119(9):2634-2647.

24. Nossent AY, Hansen JL, Doggen C, Quax PH, Sheikh SP, Rosendaal FR. SNPs in microRNA binding sites in $3^{\prime}$-UTRs of RAAS genes influence arterial blood pressure and risk of myocardial infarction. Am J Hypertens. 2011;24(9):999-1006.

25. Expert Panel on Detection, Evaluation, and Treatment of High Blood Cholesterol in Adults. Executive summary of the third report of the National Cholesterol Education Program (NCEP) Expert Panel on Detection, Evaluation, And Treatment of High Blood Cholesterol In Adults (Adult Treatment Panel III). JAMA. 2001;285(19):2486-2497.

26. Kannel WB. Risk stratification of dyslipidemia: insights from the Framingham Study. Curr Med Chem Cardiovasc Hematol Agents. 2005;3(3):187-193.

27. Fernandez ML, Webb D. The LDL to HDL cholesterol ratio as a valuable tool to evaluate coronary heart disease risk. J Am Coll Nutr. 2008;27(1):1-5.

28. Kannel WB, Dawber TR, Kagan A, Revotskie N, Stokes J 3rd. Factors of risk in the development of coronary heart disease-six year follow-up experience. The Framingham Study. Ann Intern Med. 1961; 55:33-50.

29. Castelli WP, Garrison RJ, Wilson PW, Abbott $\mathrm{RD}$, Kalousdian S, Kannel WB. Incidence of coronary heart disease and lipoprotein cholesterol levels. The Framingham Study. JAMA. 1986; 256(20):2835-2838

30. Sekine S, et al. Disruption of Dicer1 induces dysregulated fetal gene expression and promotes hepatocarcinogenesis. Gastroenterology. 2009; 136(7):2304-2315.

31. Brown MS, Goldstein JL. The SREBP pathway: regulation of cholesterol metabolism by proteolysis of a membrane-bound transcription factor. Cell. 1997;89(3):331-340.

32. Najafi-Shoushtari SH, et al. MicroRNA-33 and the SREBP host genes cooperate to control cholesterol homeostasis. Science. 2010;328(5985):1566-1569.

33. Rayner KJ, et al. MiR-33 contributes to the regulation of cholesterol homeostasis. Science. 2010; 328(5985): 1570-1573.

34. Dávalos A, et al. miR-33a/b contribute to the regulation of fatty acid metabolism and insulin signaling. Proc Natl Acad Sci U S A. 2011;108(22):9232-9237.

35. Rayner KJ, et al. Inhibition of miR-33a/b in nonhuman primates raises plasma HDL and lowers VLDL triglycerides. Nature. 2011;478(7369):404-407.
36. Lagos-Quintana M, Rauhut R, Yalcin A, Meyer J, Lendeckel W, Tuschl T. Identification of tissuespecific microRNAs from mouse. Curr Biol. 2002; 12(9):735-739.

37. Chang J, et al. miR-122, a mammalian liver-specific microRNA, is processed from hcr mRNA and may downregulate the high affinity cationic amino acid transporter CAT-1. RNA Biol. 2004;1(2):106-113.

38. $\mathrm{Xu} \mathrm{H}$, et al. Liver-enriched transcription factors regulate microRNA-122 that targets CUTL1 during liver development. Hepatology. 2010; 52(4):1431-1442.

39. Esau C, et al. miR-122 regulation of lipid metabolism revealed by in vivo antisense targeting. Cell Metab. 2006;3(2):87-98.

40. Elmén J, et al. LNA-mediated microRNA silencing in non-human primates. Nature. 2008; 452(7189):896-899.

41. Lanford RE, etal. Therapeutic silencing of microRNA122 in primates with chronic hepatitis $C$ virus infection. Science. 2010;327(5962):198-201.

42. Kannel WB, McGee DL. Diabetes and cardiovascular disease. The Framingham study. JAMA. 1979; 241(19):2035-2038.

43. Lynn FC, Skewes-Cox P, Kosaka Y, McManus MT, Harfe BD, German MS. MicroRNA expression is required for pancreatic islet cell genesis in the mouse. Diabetes. 2007;56(12):2938-2945.

44. Mudhasani R, Puri V, Hoover K, Czech MP, Imbalzano AN, Jones SN. Dicer is required for the formation of white but not brown adipose tissue. J Cell Physiol. 2011;226(5):1399-1406.

45. Poy MN, et al. A pancreatic islet-specific microRNA regulates insulin secretion. Nature. 2004; 432(7014):226-230.

46. Zhao H, et al. Up-regulated pancreatic tissue microRNA-375 associates with human type 2 diabetes through beta-cell deficit and islet amyloid deposition. Pancreas. 2010;39(6):843-846.

47. Poy MN, et al. miR-375 maintains normal pancreatic alpha- and beta-cell mass. Proc Natl Acad Sci US A. 2009;106(14):5813-5818.

48. Herrera BM, et al. Global microRNA expression profiles in insulin target tissues in a spontaneous rat model of type 2 diabetes. Diabetologia. 2010; 53(6):1099-1109.

49. Trajkovski M, et al. MicroRNAs 103 and 107 regulate insulin sensitivity. Nature. 2011;474(7353):649-653.

50 . Jordan SD, et al. Obesity-induced overexpression of miRNA-143 inhibits insulin-stimulated AKT activation and impairs glucose metabolism. Nat Cell Biol. 2011;13(4):434-446.

51. Reinhart BJ, et al. The 21-nucleotide let-7 RNA regulates developmental timing in Caenorhabditis elegans. Nature. 2000;403(6772):901-906.

52. Zhu H, et al. Lin28a transgenic mice manifest size and puberty phenotypes identified in human genetic association studies. Nat Genet. 2010; 42(7):626-630.

53. Zhu H, et al. The Lin28/let-7 axis regulates glucose 
metabolism. Cell. 2011;147(1):81-94.

54. Frost RJ, Olson EN. Control of glucose homeostasis and insulin sensitivity by the Let-7 family of microRNAs. Proc Natl Acad Sci U S A. 2011; 108(52):21075-21080.

55. Fang ZY, Prins JB, Marwick TH. Diabetic cardiomyopathy: evidence, mechanisms, and therapeutic implications. Endocr Rev. 2004;25(4):543-567.

56. van Rooij E, et al. A family of microRNAs encoded by myosin genes governs myosin expression and muscle performance. Dev Cell. 2009;17(5):662-673.

57. Callis TE, et al. MicroRNA-208a is a regulator of cardiac hypertrophy and conduction in mice. J Clin Invest. 2009;119(9):2772-2786.

58. van Rooij E, Sutherland LB, Qi X, Richardson JA, Hill J, Olson EN. Control of stress-dependent cardiac growth and gene expression by a microRNA Science. 2007;316(5824):575-579.

59. Grueter CE, et al. A cardiac microRNA governs systemic energy homeostasis by regulation of MED13. Cell. 2012;149(3):671-683.

60. Chobanian AV, Alexander RW. Exacerbation of atherosclerosis by hypertension. Potential mechanisms and clinical implications. Arch Intern Med. 1996;156(17):1952-1956.

61. Rayner KJ, et al. Antagonism of miR-33 in mice promotes reverse cholesterol transport and regression of atherosclerosis. J Clin Invest. 2011;121(7):2921-2931.

62. Cheng Y, et al. MicroRNA-145, a novel smooth muscle cell phenotypic marker and modulator, controls vascular neointimal lesion formation. Circ Res. 2009;105(2):158-166

63. Ji R, et al. MicroRNA expression signature and antisense-mediated depletion reveal an essential role of MicroRNA in vascular neointimal lesion formation. Circ Res. 2007;100(11):1579-1588.

64. Hergenreider E, et al. Atheroprotective communication between endothelial cells and smooth muscle cells through miRNAs. Nat Cell Biol. 2012; 14(3):249-256

65 . Wang S, et al. The endothelial-specific microRNA miR-126 governs vascular integrity and angiogenesis. Dev Cell. 2008;15(2):261-271.

66. Kuhnert F, et al. Attribution of vascular phenotypes of the murine Egfl7 locus to the microRNA miR-126. Development. 2008;135(24):3989-3993.

67. Zernecke A, et al. Delivery of microRNA-126 by apoptotic bodies induces CXCL12-dependent vascular protection. Sci Signal. 2009;2(100):ra81.

68. Murry CE, Jennings RB, Reimer KA. Preconditioning with ischemia: a delay of lethal cell injury in ischemic myocardium. Circulation. 1986;74(5):1124-1136.

69. Roy S, et al. MicroRNA expression in response to murine myocardial infarction: $\mathrm{miR}-21$ regulates fibroblast metalloprotease- 2 via phosphatase and tensin homologue. Cardiovasc Res. 2009; 82(1):21-29.

70. Bostjancic E, Zidar N, Glavac D. MicroRNA microarray expression profiling in human myocardial infarction. Dis Markers. 2009;27(6):255-268.

71. Aurora AB, et al. MicroRNA-214 protects the mouse heart from ischemic injury by controlling $\mathrm{Ca} 2+$ overload and cell death. J Clin Invest. 2012; 122(4):1222-1232.

72. Wang JX, et al. miR-499 regulates mitochondrial dynamics by targeting calcineurin and dynaminrelated protein-1. Nat Med. 2011;17(1):71-78.

73. Olson EN, Williams RS. Calcineurin signaling and muscle remodeling. Cell. 2000;101(7):689-692.

74. Shieh JT, Huang Y, Gilmore J, Srivastava D. Elevated miR-499 levels blunt the cardiac stress response. PLoS One. 2011;6(5):e19481.

75. Dorn GW 2nd, Matkovich SJ, Eschenbacher WH, Zhang Y. A human 3' miR-499 mutation alters cardiac mRNA targeting and function. Circ Res. 2012; 110(7):958-967.

76. da Costa Martins PA, et al. Conditional dicer gene deletion in the postnatal myocardium provokes spontaneous cardiac remodeling. Circulation. 2008; 118(15):1567-1576

77. Rao PK, et al. Loss of cardiac microRNA-mediated regulation leads to dilated cardiomyopathy and heart failure. Circ Res. 2009;105(6):585-594.

78. van Rooij E, et al. A signature pattern of stressresponsive microRNAs that can evoke cardiac hypertrophy and heart failure. Proc Natl Acad Sci U S A. 2006;103(48):18255-18260.

79. Thum T, et al. MicroRNAs in the human heart: a clue to fetal gene reprogramming in heart failure. Circulation. 2007;116(3):258-267.

80. Thum T, et al. MicroRNA-21 contributes to myocardial disease by stimulating MAP kinase signalling in fibroblasts. Nature. 2008;456(7224):980-984.

81. Patrick DM, et al. Stress-dependent cardiac remodeling occurs in the absence of microRNA-21 in mice. J Clin Invest. 2010;120(11):3912-3916

82. da Costa Martins PA, et al. MicroRNA-199b targets the nuclear kinase Dyrk1a in an auto-amplification loop promoting calcineurin/NFAT signalling. Nat Cell Biol. 2010;12(12):1220-1227.

83. Montgomery RL, et al. Therapeutic inhibition of miR-208a improves cardiac function and survival during heart failure. Circulation. 2011; 124(14):1537-1547.

84. van Rooij E, et al. Dysregulation of microRNAs after myocardial infarction reveals a role of miR-29 in cardiac fibrosis. Proc Natl Acad Sci U S A. 2008; 105(35):13027-13032.

85. Maegdefessel L, et al. Inhibition of microRNA-29b reduces murine abdominal aortic aneurysm development. J Clin Invest. 2012;122(2):497-506.

86. Boon RA, et al. MicroRNA-29 in aortic dilation: implications for aneurysm formation. Circ Res. 2011; 109(10):1115-1119.

87. Kikuchi K, et al. Primary contribution to zebrafish heart regeneration by gata $4(+)$ cardiomyocytes. Nature. 2010;464(7288):601-605

88. Porrello ER, et al. Transient regenerative potential of the neonatal mouse heart. Science. 2011; 331(6020):1078-1080.

89. Jopling C, Sleep E, Raya M, Martí M, Raya A, Izpisúa Belmonte JC. Zebrafish heart regeneration occurs by cardiomyocyte dedifferentiation and proliferation. Nature. 2010;464(7288):606-609.

90. Porrello ER, et al. MiR-15 family regulates postnatal mitotic arrest of cardiomyocytes. Circ Res. 2011; 109(6):670-679.

91. Hullinger TG, et al. Inhibition of miR-15 protects against cardiac ischemic injury. Circ Res. 2012; 110(1):71-81

92. Kent OA, Mendell JT. A small piece in the cancer puzzle: microRNAs as tumor suppressors and oncogenes. Oncogene. 2006;25(46):6188-6196.

93. Viswanathan SR, et al. Lin 28 promotes transformation and is associated with advanced human malignancies. Nat Genet. 2009;41(7):843-848.

94. Esquela-Kerscher A, et al. The let -7 microRNA reduces tumor growth in mouse models of lung cancer. Cell Cycle. 2008;7(6):759-764.

95. Wang WC, Juan AH, Panebra A, Liggett SB. MicroRNA let-7 establishes expression of beta2adrenergic receptors and dynamically down-regulates agonist-promoted down-regulation. Proc Natl Acad Sci U S A. 2011;108(15):6246-6251.

96. Fichtlscherer S, Zeiher AM, Dimmeler S. Circulating microRNAs: biomarkers or mediators of cardiovascular diseases? Arterioscler Thromb Vasc Biol. 2011; 31(11):2383-2390.

97. Santaris Pharma A/S. Multiple Ascending Dose Study of Miravirsen in Treatment-Naïve Chronic Hepatitis C Subjects. NIH Web site. http://clinicaltrials.gov/ct2/show/record/NCT01200420. Updated January 26, 2012. Accessed May 18, 2012. 